\title{
A New Wavelet Based SVM Classifier for Wild Fire Detection Using Decision Fusion Framework in Video
}

\author{
S.R Raji and Radha Krishnan B.L
}

\begin{abstract}
There has been an increasing interest in the study of video based fire detection algorithms as video based surveillance systems become widely available for indoor and outdoor monitoring applications. Although many video based smoke-detection algorithms have been developed and applied in various experimental or real life applications, but the standard method for evaluating their quality has not yet been proposed. In this framework, it is assumed that the compound algorithm consists of several subalgorithms, each of which yields its own decision as a real number centered around zero, representing the confidence level of that particular subalgorithm. In this project, the wavelet support vector machine (WSVM)-based model is used for Wild fire detection (WFD). Decision values are linearly combined with weights that are updated online according to an active fusion method based on performing entropic projections onto convex sets describing subalgorithms. The new wavelet kernel is proposed to improve the generalization ability of the support vector machine (SVM). More-over, the proposed model utilizes the principle of wavelet analysis to facilitate nonlinear characteristic extraction of the image data. To reduce misclassification due to fog, an efficient fog removal scheme using adaptive normalization method.
\end{abstract}

Index Terms-Active fusion, wildfire detection using video, Smoke detection, Wavelets Support vector machine, Video processing.

\section{Introduction}

Forest fires can be a very severe and serious problem in regions with hot climate and extensive vegetation. Video surveillance has become a widely used tool for monitoring wild fire. It is useful in many fields such as law enforcement, security, and protection of the environment. Early detection of forest fires is very important to reducing fire damage. Flames may not be visible to the monitoring camera if the flames occur in a long distance or are obscured by obstacles like mountains or buildings. Several flame detection methods have been proposed and recently the focus has shifted to smoke detection. Smoke is a good indicator of a forest fire, but it can be difficult to identify smoke in images because it does not have a specific shape or color patterns. Most of the flame detection systems are either based on pixel intensity recognition or on motion detection.

In this framework, it is assumed that the compound algorithm consists of several subalgorithms, each of which yields its own decision. The final decision is reached based on a set of real numbers representing confidence levels of various subalgorithms. Decision values are linearly combined with weights that are updated online using an active fusion method based on performing entropic projections (e-projections) onto convex sets describing the subalgorithms. A multiple-classifier system is useful for difficult pattern recognition problems, particularly when large class sets and noisy data are involved, by allowing the use of arbitrary feature descriptors and classification procedures at the same time.

A new wavelet kernel is proposed to improve the generalization ability of the support vector machine (SVM). To reduce misclassification due to fog, an efficient fog removal scheme using adaptive normalization method is used. The new wavelet kernel can vary among different kernels according to specific applications, which makes the WSVM acquire better generalization ability than the SVM with an RBF kernel. Moreover, the proposed model utilizes the principle of wavelet analysis to facilitate nonlinear characteristic extraction of the image data. Therefore, the proposed WSVM-based model [1] is superior to the conventional method. The support vector machine (SVM) is a new universal learning machine, which is applied to both regression and pattern recognition. An SVM uses a device called kernel mapping to map the data in input space to a highdimensional feature space in which the problem becomes linearly separable. The decision function of an SVM is related not only to the number of SVs and their weights, but also to the a priori chosen kernel that is called as support vector kernel.

It is valuable to study the problem of whether a better performance could be obtained if the wavelet technique is combined with SVMs. An admissible SV kernel, which is a wavelet kernel constructed in this project. It implements the combination of the wavelet technique with SVMs. The wavelet kernel has the same expression as a multidimensional wavelet function; therefore, the goal of the WSVMs is to find the optimal approximation or classification in the space spanned by multidimensional wavelets or wavelet kernels. 


\section{Existing Works}

The studies in the field of collective recognition, which were started in the mid 1950s, found wide application in practice during the last decade, leading to solutions to complex large scale applied problems.

Ko et al. [2] have proposed a non-linear classification method using support vector machines and luminescence maps, showing that the method is robust in several scenarios compared to features used earlier for flame detection. Guillemant and Vicente [3] propose an algorithm based on fractals for smoke detection in forest fire scenario with impressive results. Thou-Ho et al. [4] propose a rule based system to detect smoke which is based on pixel intensity. They perform intensity based characterization of smoke. Xu et al. [5] use single stage wavelet energy and a back propagation neural network on a small dataset for smoke detection. The system requires a high processing power which is unavailable in CCD camera networks. Piccinini et al. [6] propose a Bayesian framework for smoke motion detection using the wavelet energy of $8 \times 8$ pixel block and intensity of pixels. Vezzani et al. [7] propose a similar system in the context of ViSOR repository. Yang el al. [8] propose a support vector machine based approach using motion detection as the feature to detect the smoke contour.

Recently, Yuan et al. [9] have reported a block by block approach based on chrominance and motion orientation. They propose a new fast algorithm for motion orientation estimation. However, the chrominance based methods they use have a disadvantage in their dependence on the color of smoke. Also, the motion estimation algorithm is very time consuming in the context of smoke detection. Ferrari et al. [10] have proposed a block based approach similar to the proposed approach for steam detection in oil sand mines. They use Wavelet and Hidden Markov Model for feature extraction and support vector machine for classification with very good accuracy of over $90 \%$. However, the system is fine tuned to the oil sand application. Moreover, only the steam is characterized in their approach where as this paper presents a novel algorithm for smoke detection which has the ability to detect smoke in various scenarios.

\section{Proposed System}

In this paper, the EADF framework is applied to a computer-vision-based wildfire detection problem. The system based on this method is currently being used in more than 60 forest-fire lookout towers in the Mediterranean region. The proposed automatic video-based wildfire detection algorithm is based on five subalgorithms: 1) slow moving video object detection; 2) smoke-colored region detection; 3) wavelet-transformbased region smoothness detection; 4) shadow detection and elimination; and 5) covariance-matrix-based classification. Each sub-algorithm separately decides on the existence of smoke in the viewing range of the camera. Decisions from subalgorithms are combined with the adaptive decision fusion (ADF) method. Initial weights of the subalgorithms are determined from actual forest-fire videos and test fires. They are updated by using e-projections onto hyperplanes defined by the fusion weights. It is assumed that there is an oracle monitoring the decisions of the combined algorithm. In the wildfire detection case, the oracle is a security guard. Whenever a fire is detected, the decision should be acknowledged by the security guard. The decision algorithm will also produce false alarms in practice. Whenever an alarm occurs, the system asks the security guard to verify its decision. If it is incorrect, the weights are updated according to the decision of the security guard.

Image pre-processing can significantly increase the reliability of an optical inspection. Several filter operations which intensify or reduce certain image details enable an easier or faster evaluation. Preprocessing in images are having lots of advantages. It can reduce noise and can perform image enhancements operations such as smoothening, sharpening etc.

When the input data to an algorithm is too large to be processed and it is suspected to be notoriously redundant (e.g. the same measurement in both feet and meters) then the input data will be transformed into a reduced representation set of features (also named features vector). Transforming the input data into the set of features is called feature extraction. If the features extracted are carefully chosen it is expected that the features set will extract the relevant information from the input data in order to perform the desired task using this reduced representation instead of the full size input. To reduce misclassification due to fog, an efficient fog removal scheme using adaptive normalization method is used in the preprocessing step.

\section{Compound Algorithm}

The compound algorithm be composed of M-many detection subalgorithms $D_{1}, D_{2}, \ldots D_{m}$. Upon receiving a sample input at time step $n$, each subalgorithm yields a decision value $\mathrm{D}_{\mathrm{i}}(\mathrm{x}, \mathrm{y}) £ \mathrm{R}$ centered around zero. If $\mathrm{D}_{\mathrm{i}}(\mathrm{x}, \mathrm{y})>0$, it means that the event is detected by the th subalgorithm. Otherwise, it is assumed that the event did not happen. The type of the sample input may vary depending on the algorithm. It may be an individual pixel, or an image region, or the entire image depending on the subalgorithm of the computer vision problem. 


\section{A. Detection of Slow Moving Object}

Video objects at far distances to the camera seem to move slower ( $\mathrm{px} / \mathrm{sec}$ ) in comparison to the nearby objects moving at the same speed. Assuming the camera is fixed, two background images, $\mathrm{B}^{\mathrm{fast}}(\mathrm{x}, \mathrm{n})$ and $\mathrm{B}^{\text {slow }}(\mathrm{x}, \mathrm{n})$ corresponding to the scene with different update rates, where $\mathrm{x}$ is the location of the pixel at frame number $n$. A background image $B(x, n+1)$ at time instant $n+1$ is recursively estimated from the image frame $\mathrm{I}(\mathrm{x}, \mathrm{n})$ and the background image $\mathrm{B}(\mathrm{x}, \mathrm{n})$ depends on the location of pixel $\mathrm{x}$ at frame number $\mathrm{n}$.

$\mathrm{I}(\mathrm{x}, \mathrm{n})$ represent the intensity value of the pixel at location $\mathrm{x}$ in the $\mathrm{nth}$ video frame $\mathrm{I}$, and a is a parameter between 0 and 1 . Initially, $B^{\text {fast }}(x, 0)$ and $B^{\text {slow }}(x, 0)$ can be taken as $I(x, 0)$. Background images $\mathrm{B}^{\text {fast }}(\mathrm{x}, \mathrm{n})$ and $\mathrm{B}^{\text {slow }}(\mathrm{x}, \mathrm{n})$ are updated with different update rates. If there exists a substantial difference between the two images for some period of time, then an alarm for slow moving region is raised, and the region is marked. The decision value indicating the confidence level of the first sub-algorithm is determined by the difference between background images. The decision function $\mathrm{D}_{1}(\mathrm{x}, \mathrm{n})$ will give the confidence value.

\section{B. Detection of Smoke-Colored Regions}

Whenever a slow moving region is detected, its color content is analyzed. Smoke due to forest fires is mainly composed of carbon dioxide, water vapor, carbon monoxide, particulate matter, hydrocarbons and other organic chemicals, nitrogen oxides, trace minerals and some other compounds. The grayish color of the rising plume is primarily due to water vapor and carbon particles in the output fire composition. Such regions can be identified by setting thresholds in the YUV color space. Also, luminance value of smoke regions should be high especially at the initial phases of a wildfire. On the other hand, the chrominance values should be very low in a smoke region. Confidence value corresponding to this sub-algorithm should account for these characteristics.

The decision function $\mathrm{D}_{2}(\mathrm{x}, \mathrm{n})$ takes values between 1 and -1 depending on the values of the $\mathrm{Y}(\mathrm{x}, \mathrm{n})$, $\mathrm{U}(\mathrm{x}, \mathrm{n})$ and $\mathrm{V}(\mathrm{x}, \mathrm{n})$ channel values. $\mathrm{Y}(\mathrm{x}, \mathrm{n}), \mathrm{U}(\mathrm{x}, \mathrm{n})$ and $\mathrm{V}(\mathrm{x}, \mathrm{n})$ are the luminance and chrominance values of the pixel at location $x$ of the input image frame at time step $n$, respectively. The confidence level of $D_{2}(x, n)$ is 1 if $Y(x, n)$ is below $T_{1}$. The reason that we have the threshold $T_{1}$ is to eliminate dark regions which also have low chrominance values.

\section{Detection of Rising Regions}

Wildfire smoke regions tend to rise up into the sky at the early stages of the fire. This characteristic behavior of smoke plumes is modeled with three-state Hidden Markov Models [11] (HMM). Temporal variation in row number of the upper-most pixel belonging to a slow moving region is used as a one dimensional (1-D) feature signal, $F=f(n)$, and fed to the Markov models. One of the models $\left(\lambda_{1}\right)$ corresponds to genuine wildfire smoke regions and the other one $\left(\lambda_{2}\right)$ corresponds to regions with clouds and cloud shadows. The state S1 is attained, if the row value of the upper-most pixel in the current image frame is smaller than that of the previous frame (rise-up). If the row value of the upper-most pixel in the current image frame is larger than that of the previous frame, then $\mathrm{S} 2$ is attained and this means that the region moves-down. No change in the row value corresponds to S3.

\section{Shadow Detection \& Removal}

Shadows of slow moving clouds are major source of false alarms for video based wildfire smoke detection systems. Unfortunately, shadows of clouds have very low $\mathrm{U}$ and $\mathrm{V}$ values, similar to smoke regions due to wildfires. The decision function for shadow regions are defined based on the shadow detection method. Average RGB values are calculated for slow moving regions both in the current and the background images. Let $\mathrm{S}(\mathrm{n})$ represent a slow moving region in the image I at frame number $\mathrm{n}$.

\section{E. Covariance-Matrix-Based Region Classification}

The fifth subalgorithm deals with the classification of the smoke-colored moving regions. We first obtain a mask from the intersection of the first two subalgorithms and use the obtained smoke-colored moving regions as the input to the fifth algorithm. The regions are passed as bounding boxes of the connected regions of the mask [12]. A region covariance matrix consisting of discriminative features is calculated for each region.

\section{F. Wavelet Support Vector Machine}

The support vector machine (SVM) is a new universal learning machine, which is applied to both regression and pattern recognition. A Support Vector Machine (SVM) performs classification by constructing an $N$-dimensional hyper plane that optimally separates the data into two categories. Support Vector Machine (SVM) models are a close cousin to classical multilayer perception neural networks. Using a kernel function, SVM's are an alternative training method for polynomial, radial basis function and multi-layer perception classifiers in which the weights of the network are found by solving a quadratic programming problem with 
linear constraints, rather than by solving a non-convex, unconstrained minimization problem as in standard neural network training.

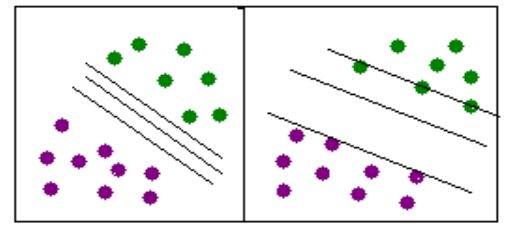

Figure 1: Group of classification

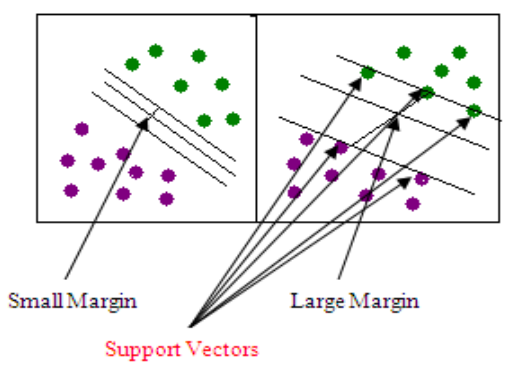

Figure 2: Margin of support vectors

The goal of the WSVMs is to find the optimal approximation or classification in the space spanned by multidimensional wavelets or wavelet kernels.

Let $h(x)$ be a mother wavelet and let $a$ and $c$ denote the dilation and translation, respectively. $x, c, a \in R$. If $\mathrm{x}, \mathrm{x} \in R^{N}$, then dot-product wavelet kernels are

$\mathrm{K}\left(\mathrm{x}, \mathrm{x}^{`}\right)=\Pi_{\mathrm{i}=1}^{\mathrm{N}} \mathrm{h}\left(\mathrm{x}_{\mathrm{i}} \mathrm{c}_{\mathrm{i}} / \mathrm{a}\right) \mathrm{h}\left(\mathrm{x}^{\prime}-\mathrm{c}_{\mathrm{i}}{ }_{\mathrm{i}} / \mathrm{a}\right)$

and translation-invariant wavelet kernels that satisfy the translation invariant kernel theorem are

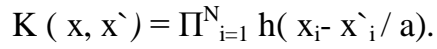

The translation-invariant wavelet kernel by a wavelet function is

$\mathrm{h}(\mathrm{x})=\cos (1.75 \mathrm{x}) \exp \left(-\mathrm{x}^{2} / 2\right)$.

The mother wavelet and the dilation $a, a, x \in R$. If $\mathrm{x}, \mathrm{x}^{`} \in R^{N}$, the wavelet kernel of this mother wavelet is

$\mathrm{K}\left(\mathrm{x}, \mathrm{x}^{`}\right)=\Pi_{\mathrm{i}=1}^{\mathrm{N}} \mathrm{h}\left(\mathrm{x}_{\mathrm{i}}-\mathrm{x}_{\mathrm{i}} / \mathrm{a}\right)$

$$
=\Pi_{i}^{\mathrm{N}}{ }_{\mathrm{i}}\left(\cos \left(1.75 \times\left(\mathrm{x}_{\mathrm{i}}-\mathrm{x}_{\mathrm{i}}{ }_{\mathrm{i}} / \mathrm{a}\right)\right) \exp \left(-\left\|\mathrm{x}_{\mathrm{i}}-\mathrm{x}_{\mathrm{i}}{ }_{\mathrm{i}}\right\|^{2} / 2 \mathrm{a}^{2}\right)\right)
$$

which is an admissible SV kernel.

From the expression of wavelet kernels, we can take them as a kind of multidimensional wavelet function. The goal of our WSVM is to find the optimal wavelet coefficients in the space spanned by the multidimensional wavelet basis.

The estimate function of WSVMs for the approximation

$f(x)=\sum_{i=1}^{1}\left(\alpha_{i}-\alpha_{i}^{*}\right) \Pi^{N}{ }_{j=1} h\left(x^{j}-x_{i}^{j} / a_{i}\right)+b$

and the decision function for classification is

$\mathrm{f}(\mathrm{x})=\operatorname{sgn}\left(\sum_{\mathrm{i}=1}^{1}\right.$ aiyi $\left.\Pi_{\mathrm{j}=1}^{\mathrm{N}} \mathrm{h}\left(\mathrm{x}^{\mathrm{j}}-\mathrm{x}_{\mathrm{i}}^{\mathrm{j}} / \mathrm{a}_{\mathrm{i}}\right)+\mathrm{b}\right)$

where the $\mathrm{x}_{\mathrm{i}}^{\mathrm{j}}$ denotes the $j$ th component of the $i$ th training example.

Decision results of five subalgorithms, $\mathrm{D}_{1}, \mathrm{D}_{2}, \mathrm{D}_{3}, \mathrm{D}_{4}$ and $\mathrm{D}_{5}$ are linearly combined by means of active decision fusion algorithm to reach a final decision on a given pixel whether it is a pixel of a smoke region or not.

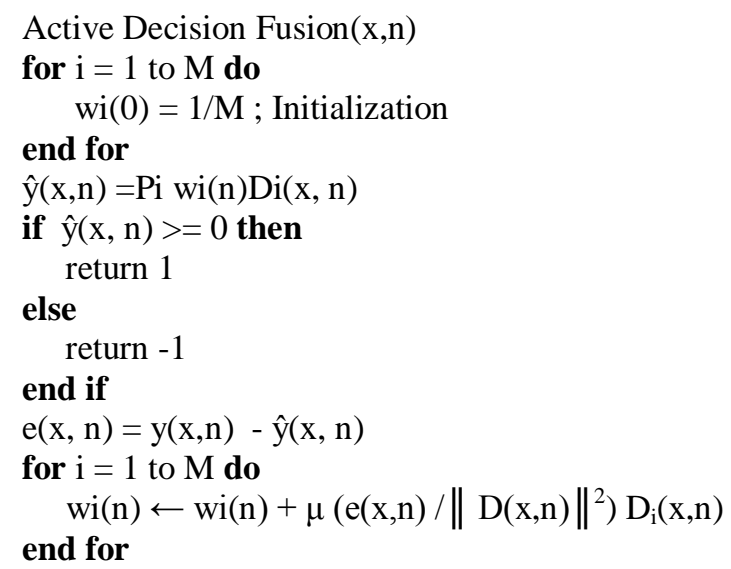


Figure 3: The pseudo-code for the active decision fusion algorithm

\section{G. Update Weight}

The main wildfire detection algorithm is composed of five subalgorithms. Each algorithm has its own decision function yielding a zero mean real number for slow moving regions at every image frame of a video sequence. Decision values from subalgorithms are linearly combined and weights of subalgorithms are adaptively updated in our approach.

The $1_{1}$-norm based minimization approaches provide successful signal reconstruction results in compressive sensing problems. However, the $1_{1}$-norm-based cost functions used in compressive sensing problems are not differentiable everywhere. The entropy functional approximates the-norm. Therefore, it can be used to find approximate $1_{1}-$ norm $\sum_{\mathrm{I}}\left|\mathrm{w}_{\mathrm{i}}(\mathrm{n})\right|$ for $\mathrm{w}_{\mathrm{i}}(\mathrm{n})>0$ solutions to the inverse problems defined and other applications requiring $1_{1}$-norm minimization. Bregman developed convex optimization algorithms in the $1960 \mathrm{~s}$, and his algorithms are widely used in many signal reconstruction and inverse problems [13]. Bregman's method provides globally convergent iterative algorithms for problems with convex, continuous, and differentiable cost functionals as follows:

$$
\min _{W € C} g(w)
$$

Such that $\mathrm{D}^{\mathrm{T}}(\mathrm{x}, \mathrm{n}) \mathrm{w}(\mathrm{n})=\mathrm{y}$ for each time index $\mathrm{n}$



Figure 4: Orthogonal Projection: Find the vector $w(n+1)$ on the hyperplane $y(x, n)=D T(x, n) w$ minimizing the distance between $\mathrm{w}(\mathrm{n})$ and the hyperplane.

In the EADF framework, the cost function is

$\mathrm{g}(\mathrm{w})=\sum_{\mathrm{i}}^{\mathrm{M}} \mathrm{w}_{\mathrm{i}}(\mathrm{n}) \log \left(\left(\mathrm{w}_{\mathrm{i}}(\mathrm{n})\right)\right.$

Let $w(n)$ denote the weight vector for the $\mathrm{n}^{\text {th }}$ sample. Its e-projection $\mathrm{w}^{*}$ onto a closed convex set $C$ with respect to a cost functional $\mathrm{g}(\mathrm{w})$ is defined as follows:

Where

$$
\mathrm{w}^{*}=\arg \min _{\mathrm{w} \in \mathrm{C}} L(\mathrm{w}, \mathrm{w}(\mathrm{n}))
$$

$L(\mathrm{w}, \mathrm{w}(\mathrm{n}))=\mathrm{g}(\mathrm{w})-\mathrm{g}(\mathrm{w}(\mathrm{n}))-<\Delta \mathrm{g}(\mathrm{w}), \mathrm{w}-\mathrm{w}(\mathrm{n})>$

The e-projection onto the hyperplane $\mathrm{H}(\mathrm{x}, \mathrm{n})$ leads to the following update equations: $\mathrm{w}_{\mathrm{i}}(\mathrm{n}+1)=\mathrm{w}_{\mathrm{i}}(\mathrm{n}) \mathrm{e}_{\mathrm{i}}^{\lambda \mathrm{D}(\mathrm{x}, \mathrm{n})}, \quad \mathrm{i}=1,2, \ldots ., \mathrm{M}$

Where the Lagrange multiplier $\lambda$ is obtained by inserting into the hyperplane equation

$\mathrm{D}^{\mathrm{T}}(\mathrm{x}, \mathrm{n}) \mathrm{w}(\mathrm{n}+1)=\mathrm{y}(\mathrm{x}, \mathrm{n})$

If the intersection of hyperplanes is an empty set, then the updated weight vector simply satisfies the last hyperplane equation [14].

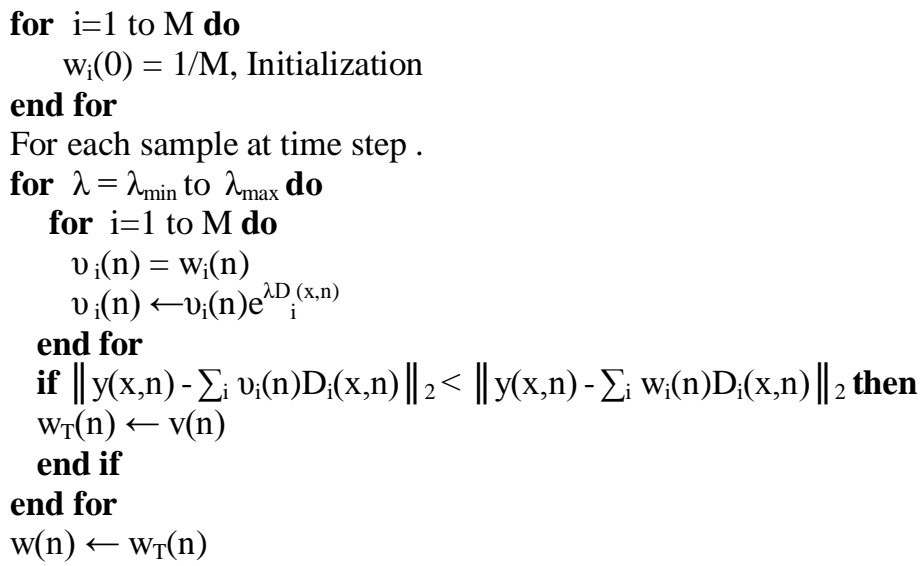


for $\mathrm{i}=1$ to $\mathrm{M}$ do

$\mathrm{w}_{\mathrm{i}}(\mathrm{n}) \leftarrow \mathrm{w}_{\mathrm{i}}(\mathrm{n}) / \sum_{\mathrm{j}} \mathrm{w}_{\mathrm{j}}(\mathrm{n})$

end for

$\hat{\mathrm{y}}(\mathrm{x}, \mathrm{n})=\mathrm{w}_{\mathrm{i}}(\mathrm{n}) \mathrm{D}_{\mathrm{i}}(\mathrm{x}, \mathrm{n})$

if $\hat{y}(x, n)>=0$ then

return 1

else

return 1

end if

Figure 5: The pseudocode for the EADF algorithm

For the wildfire detection problem, it is desirable that each subalgorithm should contribute to the compound algorithm because they characterize a feature of wildfire smoke. Therefore, weights of algorithms should be between 0 and 1 .

\section{Experimental Results}

The proposed wildfire detection schema based active learning method is compared with one of the previous smoke detection method using multiple classifier. Decision results of five subalgorithms are linearly combined by means of active decision fusion algorithm to reach a final decision on a given pixel whether it is a pixel of a smoke region or not. Results are summarized in table 1 and table 2 , in terms of the true detection rates and the miss detection rates. The true detection rate is defined as:

\begin{tabular}{|c|c|c|c|}
\hline \multicolumn{5}{|c|}{ True Detection Rates } \\
\hline Video & Frames & $\begin{array}{c}\text { Multiple } \\
\text { Classifier }\end{array}$ & WSVM \\
\hline V1 & 679 & $72.41 \%$ & $86.62 \%$ \\
\hline V2 & 300 & $78.56 \%$ & $83.45 \%$ \\
\hline V3 & 450 & $75.31 \%$ & $81.89 \%$ \\
\hline V4 & 1006 & $68.49 \%$ & $89.91 \%$ \\
\hline V5 & 785 & $74.85 \%$ & $90.01 \%$ \\
\hline V6 & 410 & $70.35 \%$ & $88.41 \%$ \\
\hline V7 & 335 & $65.32 \%$ & $81.43 \%$ \\
\hline V8 & 1000 & $72.30 \%$ & $93.07 \%$ \\
\hline V9 & 330 & $74.46 \%$ & $82.31 \%$ \\
\hline \multicolumn{5}{|c}{ Table 1 } \\
\hline
\end{tabular}

and the miss detection rate is defined as:

\begin{tabular}{|c|c|c|c|}
\hline \multicolumn{4}{|c|}{ Miss Detection Rates } \\
\hline Video & Frames & $\begin{array}{c}\text { Multiple } \\
\text { Classifier }\end{array}$ & WSVM \\
\hline V1 & 679 & $17.98 \%$ & $7.78 \%$ \\
\hline V2 & 300 & $13.34 \%$ & $6.43 \%$ \\
\hline V3 & 450 & $15.34 \%$ & $8.56 \%$ \\
\hline V4 & 1006 & $11.46 \%$ & $6.67 \%$ \\
\hline V5 & 785 & $14.82 \%$ & $8.43 \%$ \\
\hline V6 & 410 & $12.27 \%$ & $5.89 \%$ \\
\hline V7 & 335 & $9.87 \%$ & $5.32 \%$ \\
\hline V8 & 1000 & $20.34 \%$ & $9.43 \%$ \\
\hline V9 & 330 & $12.48 \%$ & $7.04 \%$ \\
\hline
\end{tabular}




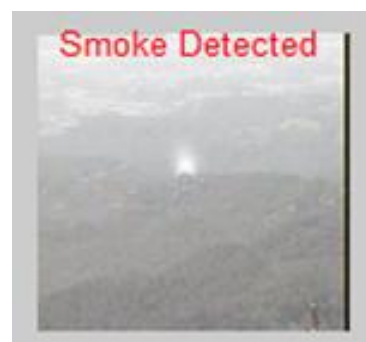

(a) Video 1

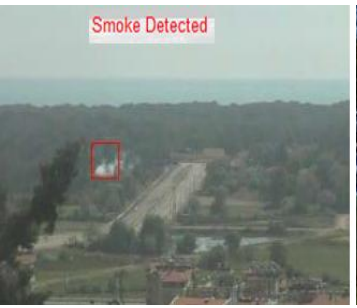

(c) Video 6

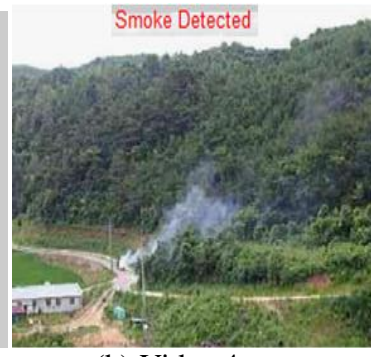

(b) Video 4

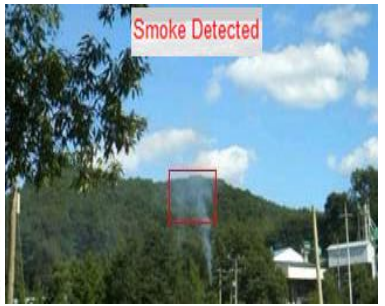

(d) Video 8

\section{Conclusion}

An EADF is proposed for image analysis and computer vision applications with drifting concepts. This general framework is applied to a real computer vision problem of wildfire detection. The proposed adaptive decision fusion strategy takes into account the feedback from guards of forest watch towers. The proposed framework for decision fusion is suitable for problems with concept drift. At each stage of the algorithm, the method tracks the changes in the nature of the problem by performing a nonorthogonal e-projection onto a hyperplane describing the decision of the oracle.

\section{References}

[1] B. U. Töreyin, Y. Dedeolu, and A. E. Çetin, "Wavelet based real-time smoke detection in video," in Proc. EUSIPCO, 2005, pp. 2 5 .

[2] B.C. Ko, K.H. Cheong, J.Y. Nam, Fire detection based on vision sensor and support vector machines, Fire Safety Journal 44 (3) (2009) 322-329..

[3] P. Guillemant, J. Vicente, Real-time identification of smoke images by clustering motions on a fractal curve with a temporal embedding method, Optical Engineering 40 (4) (2001) 554-563.

[4] C. Thou-Ho, Y. Yen-Hui, H. Shi-Feng, Y. Yan-Ting, The smoke detection for early fire-alarming system based on video processing, in: International Conference on Intelligent Information Hiding and Multimedia Signal Processing, 2006, IIH-MSP '06, 2006, pp. 427-430.

[5] Z. Xu, J. Xu, Automatic fire smoke detection based on image visual features, in: International Conference on Computational Intelligence and Security Workshops, 2007, CISW 2007, 2007, pp. 316-319.

[6] P. Piccinini, S. Calderara, R. Cucchiara, Reliable smoke detection in the domains of image energy and color, in: 15th IEEE International Conference on Image Processing, 2008, ICIP 2008, 2008, pp. 1376-1379.

[7] R. Vezzani, S. Calderara, P. Piccinini, R. Cucchiara, Smoke detection in video surveillance: the use of visor (video surveillance online repository), in: Proceedings of the 2008 International Conference on Content-based Image and Video Retrieval, Niagara Falls, Canada, ACM Press, New York, 2008.

[8] J. Yang, F. Chen,W. Zhang, Visual-based smoke detection using support vector machine, in: Fourth International Conference on Natural Computation, 2008, ICNC '08, vol. 4, 2008, pp. 301-305.

[9] F. Yuan,A fast accumulative motion orientation model based on integral image for video smoke detection, Pattern Recognition Letters 29 (2008) 925932.

[10] R.J.Ferrari, H. Zhang, C.R. Kube, Real-time detection of steam in video images, Pattern Recognition 40 (3) (2007) 1148-1159.

[11] B. U. Töreyin, Y. Dedeolu, and A. E. Çetin, "Flame detection in video using hidden Markov models," in Proc. ICIP, 2005, pp. II1230-II-1233.

[12] O. Tuzel, F. Porikli, and P. Meer, "Region covariance: A fast descriptor for detection and classification," in Proc. ECCV, 2006, pp. $589-600$.

[13] D. C. Youla and H.Webb, "Image restoration by the method of convex projections, Part I-Theory," IEEE Trans. Med. Imag., vol. MI-1, no. 2, pp. 81-94, Oct. 1982.

[14] L. G. Gubin, B. T. Polyak, and E. V. Raik, "The method of projections for finding the common point of convex sets," USSR Comput. Math. Phys., vol. 7, no. 6, pp. 1-24, 1967. 


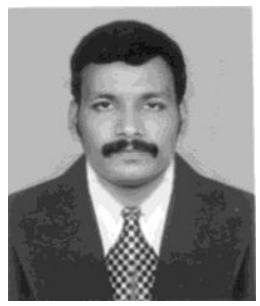

BL Radhakrishnan received his B.E. Degree in Computer Science and Engineering from Anna University, Chennai, India. In addition, he received the M.Tech degree in Computer Science and Engineering from Dr.MGR University, Chennai, India. Currently, he is an Assistant Professor in the department of Computer Science and Engineering, Marthandam College of Engineering and Technology, India. His research interests include Image Processing, Databases and Cloud Computing. He is a Life member of ISTE.

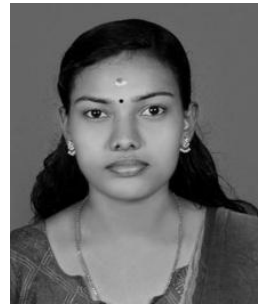

SR Raji received her B.Tech Degree in Computer Science and Engineering from Cochin University, kerala, India. Currently, she is doing her post graduate course (M.E) in the department of Computer Science and Engineering, Marthandam College of Engineering and Technology, India. Her research interests include Image Processing, Networking, Operating System and Cloud Computing. 